\title{
Detection of diverse genotypes of Methicillin-resistant Staphylococcus aureus from hospital personnel and the environment in Armenia
}

Hermine V. Mkrtchyan ${ }^{1,2^{*}}$, Zhen Xu', Maria Yacoub ${ }^{3}$, Mary M. Ter-Stepanyan ${ }^{4}$, Hayk D. Karapetyan ${ }^{4}$, Angela M. Kearns ${ }^{3}$, Ronald R. Cutler ${ }^{1}$, Bruno Pichon ${ }^{3}$ and Armen Dz Hambardzumyan ${ }^{4}$

\begin{abstract}
Background: Methicillin-resistant Staphylococcus aureus (MRSA) is a public health concern internationally. Studies examining a range of cohorts have been reported from various regions of the world, but little is known about the molecular epidemiology of MRSA in Armenia.

Methods: Between May and September 2013, twenty isolates of methicillin-resistant Staphylococcus aureus (MRSA; mecA positive) were recovered from hospital personnel ( $n=10$; 9 females, 1 male) and environmental sites $(n=10)$ in the maternity ward of one of the teaching hospitals in Armenia.

Results: Multi-locus sequence type clonal complex (MLST-CC) assignments inferred from spa typing data revealed the majority belonged to 3 pandemic lineages of MRSA including: t008-CC8-SCCmecV ( $n=10 ; 7$ from personnel); t021-CC30-SCCmedV ( $n=5$; all environmental); and t1523-CC45 ( $n=2 ; 1$ from personnel), one harboured SCCmecV the other was SCCmec non-typable. The remainder identified as belonging to genotype t364-CC182, both of which harboured a novel SCCmec cassette with kdp, rif5, ccrB2 and ccrC detected by PCR (both from personnel); and t325-CC88-SCCmedV ( $n=1$; environmental). All MRSA were negative for the Panton-Valentine Leukocidin (PVL) locus and three CC8 strains were positive for the arginine catabolic element (ACME).

Conclusions: In this small study, we report for the first time of the occurrence of diverse MRSA genotypes belonging to both pandemic and more sporadic international clones in Armenia harbouring the smaller SCCmec types and/or ACME, both of which have been associated with strain fitness. Further surveillance is warranted to better understand the prevalence, clinical and molecular epidemiology of MRSA throughout Armenia.
\end{abstract}

Keywords: MRSA, SCCmec, ACME, Pandemic, Maternity ward

\section{Background}

Methicillin-resistant Staphylococcus aureus (MRSA) is a major pathogen responsible for a wide range of mild to life threatening infections and is estimated to affect more than 150,000 patients annually in the European Union with associated costs of EUR 380 million to healthcare settings [1].

\footnotetext{
* Correspondence: h.mkrtchyan@qmul.ac.uk; h.mkrtchyan@uel.ac.uk 'Queen Mary University of London, School of Biological and Chemical Sciences, Mile End Road, London E1 4NS, UK

${ }^{2}$ School of Health, Sport and Bioscience, University of East London, Water Lane, London E15 4LZ, UK

Full list of author information is available at the end of the article
}

Reports of MRSA from an expanding range of ecological niches (healthcare, community, livestock, wildlife, environmental sources, etc.) are a public health concern internationally. Diversity in MRSA genotypes and their prevalence in different geographic areas continue to increase [2-5]. Studies examining a broad range of cohorts have been reported from various regions of the world [6-9], but little is known of the situation in some areas. Although MRSA clones from some countries have been well characterized $[8,10]$, there are few published studies describing the situation in the former USSR (Russia, Georgia), and none from Armenia [11-13]. 
The Republic of Armenia (part of the former Soviet Union) has three million inhabitants, half of whom live in the capital of Yerevan. Armenia is a low-middle income country and, currently, no population surveillance is being carried out in patients entering the hospital with the symptoms of illness compatible with staphylococcal disease. In this study, we report for the first time the molecular characterisation of MRSA recovered from hospital personnel and the environment in a University teaching hospital in the Republic of Armenia (part of the former Soviet Union). These data provide evidence for the first time of the occurrence of pandemic and more sporadic international MRSA clones in Armenia that harbour the smaller SCCmec types generally associated with strain fitness.

\section{Methods}

\section{Study protocol}

As part of a pilot surveillance study to assess the distribution and prevalence of MRSA, 450 samples were taken from hospital personnel $(n=150)$ and environmental sites $(n=300)$ in the maternity ward of one of the teaching hospitals in Armenia between May 2013 and September 2013. For hospital-based personnel (doctors, nurses and theatre nurses), samples were taken from the nasal cavity. Environmental sites included taps, patient examination chairs, surgical tables, nurse laboratory coats, baby scales, door handles and telephones. All specimens were inoculated onto nutrient agar (Oxoid, Basingstoke, UK) and incubated aerobically at $37^{\circ} \mathrm{C}$ for $24-48$ hours.

\section{Identification of the bacterial isolates}

Suspected S. aureus were initially identified using conventional methods, including growth on Mannitol Salt Agar (Oxoid Ltd, Basingstoke, UK), slide coagulase and latex agglutination testing (ProLab Diagnostics, Neston, UK). To identify possible MRSA, isolates were sub-cultured onto Chromogenic MRSA agar (Oxoid Ltd, Basingstoke, UK). Those which grew yielding characteristic blue colonies were identified further by penicillin-binding protein (PBP2') agglutination testing (Oxoid Ltd, Basingstoke, UK).

\section{Phenotypic and genotypic characterisation of MRSA}

Presumptive MRSA were screened for susceptibility to 4 antimicrobial agents (penicillin, cefoxitin, erythromycin and gentamicin) by disk diffusion and assigned as susceptible, intermediate or resistant according to the recommendations of the Clinical and Laboratory Standard Institute (CLSI) [14].

Isolates were characterised by real-time PCR to confirm they were $S$. aureus (nuc positive) and to determine their $m e c A, m e c C$ and $l u k-P V$ status, as described previously [15]. MRSA were characterised further by spa typing [16], and staphylococcal chromosomal cassette mec (SCCmec) typing [17]. Spa typing data were used to infer multi-locus sequence type clonal complex (MLST-CC) assignments by reference to the spa server (http://spa.ridom.de/mlst.shtml), MLST (http://saureus.mlst.net) and in-house (Public Health England) databases. All CC8 MRSA were screened by PCR for the presence of the ACME element [18].

\section{Results}

Over a 5 month period (May - September 2013), S. aureus was recovered from a total of 65 samples including 32 of 150 (21.3\%) hospital personnel and 33 of 300 (11\%) environmental sites in the maternity ward of one of the teaching hospitals in Armenia. Twenty (30.8\%) S. aureus identified as MRSA. Half of these $(n=10)$ were recovered from hospital personnel including 9 females and one male; the remainder were recovered from the environment (Table 1).

All MRSA were nuc and mecA positive; no mecC-MRSA were identified. All were resistant to $\beta$-lactams (penicillin and cefoxitin); three were also resistant to erythromycin and six showed intermediate resistance (zone sizes 20$22 \mathrm{~mm}$ ); in addition, one MRSA showed intermediate resistance to gentamicin (zone size $14 \mathrm{~mm}$ ) (Table 1).

Most (17; 85\%) study isolates belonged to pandemic genotypes of MRSA (Table 1), specifically: t008-CC8SCCmecV $(n=10)$; t021-CC30-SCCmecIV $(n=5)$; t1523$\mathrm{CC} 45$, one of which harboured SCCmecV, the other was SCCmec non-typable. The remainder belonged to more sporadic international lineages including t364-CC182harbouring a novel SCCmec cassette with $k d p$, rif5, $c c r B 2$ and $c c r C$ detected by PCR $(n=2)$; and t325-CC 88 SCCmecIV $(n=1)$. All MRSA were PVL negative; three CC8 strains were ACME positive (Table 1).

\section{Discussion}

The spread of antimicrobial resistant clones such as MRSA not only in healthcare and community settings but also livestock and companion animals is a major public health concern world-wide. In developing countries, the broader public health impact is worrisome due to the extensive and uncontrolled use of antimicrobial agents [7].

The aim of this study was to evaluate the clonal diversity, virulence and antibiotic susceptibility profiles of MRSA recovered from personnel and the environment in a University teaching hospital in the Republic of Armenia.

Twenty (30.8\%) out of $65 \mathrm{~S}$. aureus isolates recovered in this study were identified as MRSA. Various MRSA clones have been described globally, including some from the post-soviet countries [11-13, 19-21], however, little is known about MRSA in Armenia. The international ST239 clone has been reported as being dominant in Krasnoyarsk, Vladivostock and Georgia [11-13]. During the course of this small scale study we did not find evidence of this clone. Nevertheless, other international lineages were identified and a marked genetic 
Table 1 Susceptibility profiles and molecular characterisation of MRSA recovered from hospital personnel and environmental sites in the maternity wards of one of the teaching hospitals in Armenia

\begin{tabular}{|c|c|c|c|c|c|c|c|c|c|}
\hline No & Source & $P G$ & FOX & GM & ERY & Spa type & Inferred MLST-CC & SCCmec types & ACME \\
\hline 1 & $E$ & $\mathrm{R}$ & $R$ & $\mathrm{~s}$ & $\mathrm{I}^{\mathrm{a}}$ & t021 & 30 & IV & ND \\
\hline 24 & E & $\mathrm{R}$ & $R$ & $1^{a}$ & R & t021 & 30 & IV & ND \\
\hline 26 & E & $\mathrm{R}$ & $\mathrm{R}$ & S & S & t021 & 30 & IV & ND \\
\hline 30 & E & $\mathrm{R}$ & $\mathrm{R}$ & S & $\mathrm{R}$ & t021 & 30 & IV & ND \\
\hline 203 & $P$ & $\mathrm{R}$ & $\mathrm{R}$ & S & $1^{a}$ & t364 & $182^{a}$ & Novel $^{b}$ & ND \\
\hline 210 & P & $\mathrm{R}$ & R & S & S & t364 & $182^{\mathrm{a}}$ & Novel $^{\text {b }}$ & ND \\
\hline 221 & E & $\mathrm{R}$ & $\mathrm{R}$ & S & $1^{a}$ & t021 & 30 & IV & ND \\
\hline 222 & $P$ & $\mathrm{R}$ & $\mathrm{R}$ & S & $1^{a}$ & t008 & 8 & V & - \\
\hline 223 & $P$ & $\mathrm{R}$ & $\mathrm{R}$ & $S$ & S & t008 & 8 & V & + \\
\hline 226 & $P$ & $\mathrm{R}$ & $\mathrm{R}$ & $S$ & S & $\mathrm{t} 1523$ & 45 & V & ND \\
\hline 227 & P & $\mathrm{R}$ & $\mathrm{R}$ & S & S & t008 & 8 & V & - \\
\hline 229 & $E$ & $\mathrm{R}$ & $\mathrm{R}$ & S & S & t008 & 8 & V & + \\
\hline 230 & $E$ & $\mathrm{R}$ & R & $S$ & S & t1523 & 45 & Non-typable & ND \\
\hline 231 & $E$ & $\mathrm{R}$ & R & S & S & t008 & 8 & V & - \\
\hline 232 & $E$ & $\mathrm{R}$ & R & S & $\mathrm{R}$ & t008 & 8 & V & + \\
\hline 233 & $E$ & R & $\mathrm{R}$ & $S$ & S & t325 & 88 & IV & ND \\
\hline 236 & P & $\mathrm{R}$ & R & S & 1 & t008 & 8 & V & - \\
\hline 238 & $P$ & $\mathrm{R}$ & $\mathrm{R}$ & S & S & t008 & 8 & V & - \\
\hline 244 & P & $\mathrm{R}$ & R & S & I & t008 & 8 & V & - \\
\hline 245 & $P$ & $\mathrm{R}$ & $\mathrm{R}$ & $S$ & S & t008 & 8 & V & - \\
\hline
\end{tabular}

$P$ personnel, $E$ environment, $P G$ penicillin, FOX cefoxitin, GM gentamicin, ERY erythromycin, Spa staphylococcal protein A, MLST-CC Multi-locus sequence type clonal complex, SCCmec staphylococcal cassette chromosome mec, ACME arginine catabolic mobile element, ND not determined, + positive, - negative, all isolates were nuc and mecA positive, and luk-PV negative

${ }^{\text {a }}$ Singletons that do not fall into a clonal complex (CC) described in the S. aureus database (Skramm et al., 2007)

${ }^{\mathrm{b}} \mathrm{kdp}$, rif5, ccrB2 and $c \mathrm{crC}$ detected by PCR

$R$ resistant, $S$ susceptible, ${ }^{\mathrm{a}}$ intermediate resistance. Zone sizes for intermediate resistance were ${ }^{\mathrm{a}} \mathrm{ERY} 20-22 \mathrm{~mm}$; ${ }^{\mathrm{a}} \mathrm{Gen} 14 \mathrm{~mm}(\mathrm{Cockerill} \mathrm{FR}$, [14])

diversity was apparent. The CC8-V lineage was predominant $(n=10 ; 50 \%)$ and was identified in both human and environmental sources. Three of $10 \mathrm{CC} 8 \mathrm{-V}$ isolates were recovered from the hospital personnel, the remainder were from the environment, which may reflect crosscontamination between personnel and the environment (Table 1). All CC8-V isolates were resistant to penicillin and cefoxitin; four also showed non-susceptibility to erythromycin. Distinct from the pandemic CC8-IV MRSA lineage associated with both healthcare- and communityassociated infections [22, 23], CC8-V MRSA have been reported more sporadically [24]. In contrast to most reports of CC8-MRSA from various regions of the world (including Russia and Europe) that encode SCCmecIV [13, 25-29], all CC8 isolates in our study encoded $\mathrm{SCCmecV}$ and 3 of 10 were ACME positive. Aside from the successful USA300 (CC8-IV) clone of CA-MRSA in North America, the ACME element has been identified in a limited number of MRSA genotypes including ST5-II, ST59-IV, ST97-V, ST1-IV, ST5-IV and ST239-III [23, 30]. Interestingly, CC8-V has been found sporadically in Australia and Africa [24] but, to our knowledge, this is the first report of ACME in this lineage. Of note, the first case of CA-MRSA infection in Portugal caused by an ST8, spa type t008 strain was recovered from a male of Armenian ethnicity [31]. However, the isolate encoded SCCmecIV which differs from the $\mathrm{CC} 8 \mathrm{-V}$ isolates identified in this study.

The second most common lineage identified was CC30IV. CC30 is a widely disseminated pandemic clone, that has been associated with HA-MRSA, CA-MRSA and LAMRSA [32]. In this study all $(n=5)$ CC30-IV isolates were recovered from the environment (Table 1). Whilst the pandemic HA-MRSA lineage encodes SCCmecII (ST36-II; UK EMRSA-16 clone), PVL-negative CC30-IV MRSA strains have been reported in countries such as Ireland [33] and Australia [32].

Two isolates belonged to CC45, spa type t1523 one with $\mathrm{SCCmecV}$, the other was SCCmec non-typable. CC45 has is predominantly been associated with SCCmec type IV, which is also known as Berlin Epidemic strain or USA 600 [32]. However, CC45-MRSA-V strains have been reported in Germany, Australia and Portugal [32, 34]. Two 
isolates belonged to $\mathrm{CC} 182$; this clonal complex has been reported as a singleton [35] and MRSA belonging to CC182 have occasionally been identified in the UK and the Netherlands (http://spa.ridom.de/index.shtml).

In the current study we also identified a single CC88-IV strain. The CC88 lineage is prevalent among MRSA isolates from Africa [36] but has also been reported in Australia, Germany [32] the Netherlands, Portugal, Angola and Japan [33]. Interestingly, CC45-t1523 isolates $(n=2)$ were recovered from both hospital personnel and the environment, whereas CC182-t364 were isolated from the personnel only and CC88-t325 were identified from the environment only (Table 1). All 20 MRSA in our study were PVL-negative. This is consistent with the observations of other workers that PVL-positive MRSA is less prevalent in Europe than in the USA [32, 37]. As there are large Armenian communities in the US, Europe and Russia with relevant family links in Armenia it seems plausible that these clones were imported into Armenia from abroad in parallel with exchange of mobile genetic elements within the staphylococcal gene pool such as SCCmec and ACME.

\section{Conclusions}

There are clear limitations in this small scale study. Clinical and epidemiological data were lacking; it is unclear whether any of the isolates may have been outbreak related or there were underlying risk factors such as previous healthcare contact or travel abroad. Similarly, we do not know if any isolates were multiply resistant as only a limited range of susceptibilities were determined. Nevertheless, this study provides insights into a previously unrecognised diversity of MRSA clones in Armenia including pandemic and more sporadic lineages seen internationally. These data also provide evidence that MRSA with a community-like genotype may be infiltrating healthcare settings in this country. In low and middle income countries healthcareand community-associated infections are more challenging due to the lack of effective antimicrobial stewardship allied to infection control and prevention programmes [38]. Currently, no MRSA infection control programme exists in Armenia and no formal surveillance is being carried out in patients admitted to hospital with recognised risk factors, signs or symptoms compatible with MRSA/ staphylococcal disease. Additional studies are warranted to further our understanding of the prevalence and molecular epidemiology of MRSA in healthcare settings in Armenia. In particular, we plan a more structured surveillance study of patients and hospital personnel with more detailed analyses to further our understanding of possible risk factors, burden of disease, genetic diversity and antimicrobial resistance rates to help inform national policy.

\section{Abbreviations}

ACME: Arginine catabolic mobile element; CLSI: Clinical and Laboratory Standard Institute; ERY: Erythromycin; FOX: Cefoxitin; GM: Gentamicin; MLST-CC: Multi-locus sequence type clonal complex; MRSA: Methicillinresistant Staphylococcus aureus; PG: Penicillin; PVL: Panton-Valentine Leukocidin; SCCmec: Staphylococcal cassette chromosome mec

Acknowledgements

Not applicable.

\section{Funding}

The pilot study leading to this manuscript was funded by the Society of Applied Microbiology.

\section{Availability of data and materials}

All data generated or analysed during this study are included in this published article.

\section{Authors' contributions}

HVM study design, laboratory work, data analysis, manuscript preparation; ZX laboratory work, manuscript preparation; MY laboratory work; MMTS laboratory work; HDK laboratory work; AMK data analysis, manuscript preparation; RRC study design; BP data analysis, manuscript preparation; ADH study design, laboratory work. All authors read and approved the final manuscript.

\section{Competing interests}

The authors declare that they have no competing interests.

Consent for publication

Not applicable.

Ethics approval and consent to participate

Not applicable.

\section{Author details}

${ }^{1}$ Queen Mary University of London, School of Biological and Chemical Sciences, Mile End Road, London E1 4NS, UK. ${ }^{2}$ School of Health, Sport and Bioscience, University of East London, Water Lane, London E15 4LZ, UK. ${ }^{3}$ Antimicrobial Resistance and Healthcare Associated Infections Reference Unit, Public Health England, National Infection Service, Colindale, London, UK. ${ }^{4}$ Yerevan State Medical University, Yerevan, Armenia.

Received: 23 August 2016 Accepted: 3 January 2017

Published online: 07 February 2017

\section{References}

1. Köck R, Becker K, Cookson B, van Gemert-Pijnen JE, Harbarth S, Kluytmans J, et al. Methicillin-resistant Staphylococcus aureus (MRSA): burden of disease and control challenges in Europe. Euro Surveill. 2010;15(41).

2. Larsen AR, Stegger M, Böcher S, Sørum M, Monnet DL, Skov RL. Emergence and characterization of community-associated methicillin-resistant Staphyloccocus aureus infections in Denmark, 1999 to 2006. J Clin Microbiol. 2009:47:73-8.

3. Scribel LV, Silva-Carvalho MC, Souza RR, Superti SV, Kvitko CHC, Figueiredo AMS, et al. Clinical and molecular epidemiology of methicillin-resistant Staphylococcus aureus carrying SCCmecIV in a university hospital in Porto Alegre, Brazil. Diagn Microbiol Infect Dis. 2009;65:457-61.

4. Vandenesch F, Naimi T, Enright MC, Lina G, Nimmo GR, Heffernan H, et al. Community-acquired methicillin-resistant Staphylococcus aureus carrying Panton-Valentine leukocidin genes: worldwide emergence. Emerg Infect Dis. 2003;9:978-84.

5. Verkade E, Kluytmans J. Livestock-associated Staphylococcus aureus CC398: animal reservoirs and human infections. Infect Genet Evol. 2014;21:523-30.

6. Al-Abdli NE, Baiu SH. Isolation of MRSA Strains from Hospital Environment in Benghazi City, Libya. Am J Infect Dis Microbiol. 2016;4:41-3.

7. Alesana-Slater J, Ritchie S. Methicillin-resistant staphylococcus aureus, Samoa, 2007-2008. Emerg Infect Dis. 2011;17:1023-9.

8. Blumental S, Deplano A, Jourdain S, De Mendonça R, Hallin M, Nonhoff C, et al. Dynamic pattern and genotypic diversity of Staphylococcus aureus nasopharyngeal carriage in healthy pre-school children. J Antimicrob Chemother. 2013;68:1517-23. 
9. Faires MC, Pearl DL, Berke O, Reid-Smith RJ, Weese JS. The identification and epidemiology of meticillin-resistant Staphylococcus aureus and Clostridium difficile in patient rooms and the ward environment. BMC Infect Dis. 2013;13:1.

10. Ruppe E, Barbier F, Mesli Y, Maiga A, Cojocaru R, Benkhalfat M, et al. Diversity of Staphylococcal Cassette Chromosome mec Structures in Methicillin-Resistant Staphylococcus epidermidis and Staphylococcus haemolyticus Strains among Outpatients from Four Countries. Antimicrob Agents Chemother. 2008;53:442-9.

11. Baranovich T, Zaraket H, Shabana II, Nevzorova V, Turcutyuicov V, Suzuki H. Molecular characterization and susceptibility of methicillin-resistant and methicillin-susceptible Staphylococcus aureus isolates from hospitals and the community in Vladivostok, Russia. Clin Microbiol Infect. 2010;16:575-82.

12. Bartels MD, Nanuashvili A, Boye K, Rohde SM, Jashiashvili N, Faria NA, et al. Methicillin-resistant Staphylococcus aureus in hospitals in Tbilisi, the Republic of Georgia, are variants of the Brazilian clone. Eur J Clin Microbiol Infect Dis. 2008;27:757-60.

13. Khokhlova OE, Hung W-C, Wan T-W, Iwao Y, Takano T, Higuchi W, et al. Healthcare-and Community-Associated Methicillin-Resistant Staphylococcus aureus (MRSA) and Fatal Pneumonia with Pediatric Deaths in Krasnoyarsk, Siberian Russia: Unique MRSA's Multiple Virulence Factors, Genome, and Stepwise Evolution. PLoS One. 2015;10:e0128017.

14. Cockerill FR. Performance standards for antimicrobial susceptibility testing : twenty-third informational supplement. 2013. Clinical and Laboratory Standards Institute.

15. Pichon B, Hill R, Laurent F, Larsen AR, Skov RL, Holmes M, et al. Development of a real-time quadruplex PCR assay for simultaneous detection of nuc, PantonValentine leucocidin (PVL), mecA and homologue mecA $A_{L G A 251}$. J Antimicrob Chemother. 2012;67:2338-41.

16. Harmsen D, Claus H, Witte W, Rothgänger J, Claus H, Turnwald D, et al. Typing of methicillin-resistant Staphylococcus aureus in a university hospital setting by using novel software for spa repeat determination and database management. J Clin Microbiol. 2003;41:5442-8.

17. Milheirico C, Oliveira DC, de Lencastre H. Update to the Multiplex PCR Strategy for Assignment of mec Element Types in Staphylococcus aureus. Antimicrob Agents Chemother. 2007;51:3374-7.

18. Diep BA, Gill SR, Chang RF, Phan TH, Chen JH, Davidson MG, et al. Complete genome sequence of USA300, an epidemic clone of community-acquired meticillin-resistant Staphylococcus aureus. Lancet. 2006;367:731-9.

19. Yamamoto T, Takano T, Higuchi W, Iwao Y. Comparative genomics and drug resistance of a geographic variant of ST239 methicillin-resistant Staphylococcus aureus emerged in Russia. PLoS One. 2012;7:e29187.

20. David M, Boyle-Vavra S, Zychowski D, Daum R. Methicillin-susceptible Staphylococcus aureus as a predominantly healthcare-associated pathogen: a possible reversal of roles. PLoS One. 2011;6:e18217.

21. Nimmo GR. USA300 abroad: global spread of a virulent strain of community-associated methicillin-resistant Staphylococcus aureus. Clin Microbiol Infect Dis. 2012;18:725-34

22. Jiménez JN, Ocampo AM, Vanegas JM, Rodriguez EA, Mediavilla JR, Chen L, et al. CC8 MRSA strains harboring scc mec type ivc are predominant in Colombian hospitals. PLoS One. 2012;7:e38576.

23. Sowash M, Uhlemann A. Community-associated methicillin-resistant Staphylococcus aureus case studies. Methicillin-Resistant Staphylococcus Aureus Protoc. 2014;1085:25-69.

24. Chung M, Ch K k, Conceição T, Aires-De-Sous M, De Lencastre H, Tomasz A. Heterogeneous oxacillin-resistant phenotypes and production of PBP2A by oxacillin-susceptible/mecA-positive MRSA strains from Africa. J Antimicrob Chemother. 2016;71:2804-9.

25. Monecke S, Berger-Bächi B, Coombs G, Holmes A, Kay I, Kearns A, et al. Comparative genomics and DNA array-based genotyping of pandemic Staphylococcus aureus strains encoding Panton-Valentine leukocidin. Clin Microbiol Infect. 2007;13:236-49.

26. Grundmann $H$, Aanensen DM, Van Den Wijngaard CC, Spratt BG, Harmsen D, Friedrich AW, et al. Geographic distribution of Staphylococcus aureus causing invasive infections in Europe: a molecular-epidemiological analysis. PLoS Med. 2010;7:e1000215.

27. Kinnevey P, Shore A, Rossney A, Coleman D. Molecular characterization of sporadically-occurring nosocomial methicillin-resistant Staphylococcus aureus isolates from Ireland. Vienna: ECCMID; 2010.

28. Sara C, Golding GR, Jennifer C, Mulvey MR. Comparative genomics of Canadian epidemic lineages of methicillin-resistant Staphylococcus aureus. J Clin Microbiol. 2007:45:1904-11.
29. Weber S, Ehricht R. Slickers P. ECCMID: Genetic fingerprinting of MRSA from Abu Dhabi; 2010.

30. Shore AC, Deasy EC, Slickers P, Brennan G, O'Connell B, Monecke S, et al. Detection of Staphylococcal Cassette Chromosome mec Type XI Carrying Highly Divergent mecA, mecl, mecR1, blaZ, and ccr Genes in Human Clinical Isolates of Clonal Complex 130 Methicillin-Resistant Staphylococcus aureus. Antimicrob Agents Chemother. 2011;55:3765-73.

31. Nazareth R, Gonçalves-Pereira J, Tavares A, Miragaia M, De LH, Silvestre J, et al. Community-associated methicillin-resistant Staphylococcus aureus infection in Portugal. Rev Port Pneumol. 2012;18:34-8.

32. Monecke S, Coombs G, Shore AC, Coleman DC, Akpaka P, Borg M, et al. A field guide to pandemic, epidemic and sporadic clones of methicillinresistant Staphylococcus aureus. PLoS One. 2011;6:e17936.

33. Shore A, Rossney A. Seven novel variants of the staphylococcal chromosomal cassette mec in methicillin-resistant Staphylococcus aureus isolates from Ireland. Antimicrob Agents Chemother. 2005;49:2070-83.

34. Aires-de-Sousa M, de Lencastre $H$, the Multilaboratory Project Collaborators BC. Changing Patterns in Frequency of Recovery of Five Methicillin-Resistant Staphylococcus aureus Clones in Portuguese Hospitals: Surveillance over a 16-Year Period. J Clin Microbiol. 2008:46:2912-7.

35. Skråmm I, Moen AEF, Alm-Kristiansen K, Bukholm G. Nasal carriage of Staphylococcus aureus: which sequence types do orthopedic surgical healthcare workers carry? Infect Control Hosp Epidemiol. 2007;28:737-9.

36. Schaumburg F, Alabi AS, Peters G, Becker K. New epidemiology of Staphylococcus aureus infection in Africa. Clin Microbiol Infect. 2014;20:589-96.

37. Rossney A, Shore A. The emergence and importation of diverse genotypes of methicillin-resistant Staphylococcus aureus (MRSA) harboring the PantonValentine leukocidin gene (pvl). J Clin Microbiol. 2007;45:2554-63.

38. Shears P. Poverty and infection in the developing world: Healthcare-related infections and infection control in the tropics. J Hosp Infect. 2007:67:217-24.

\section{Submit your next manuscript to BioMed Central and we will help you at every step:}

- We accept pre-submission inquiries

- Our selector tool helps you to find the most relevant journal

- We provide round the clock customer support

- Convenient online submission

- Thorough peer review

- Inclusion in PubMed and all major indexing services

- Maximum visibility for your research

Submit your manuscript at www.biomedcentral.com/submit
) Biomed Central 